Sonderabdruck

aus ,Zeitschrift für Lebensmittel-Untersuchung und -Forschung ${ }^{\text {" }}$

126. Band, 5. Heft, 1965, Seite 344-356

J. F. Bergmann, München /Springer-Verlag, Berlin Heidelberg New York

\title{
Probleme um die Gefriertrocknung von Lebensmitteln*
}

Von

G. Kaluistratos und R. von Sengbusch

Mitteilung aus dem Max-Planck-Institut für Kulturpflanzenzïchtung Hamburg-Volksdorf

Mit 2 Textabbildungen

(Eingegangen am 23. April 1964)

* Diese Arbeit wurde von der Deutschen Forschungsgemeinschaft finanziell gefördert. 
Vorbemerkungen

Seit einer Reihe von Jahren gesellt sich zu den bekannten Konservierungsverfahren von Obst und Gemüse ein neues Verfahren, das der Gefriertrocknung $(1,2,3)$. Um die Vor- und Nachteile dieses Verfahrens zu erkennen, ist es notwendig, die einzelnen Konservierungsverfahren vergleichend zu betrachten. 


\section{Vergleich der Konservierungsarten}

Man kann bei der Konservierung von Obst und Gemüse nach den verschiedenen Konservierungsverfahren eine unterschiedliche Abnahme der ursprünglichen Qualität feststellen. Einerseits findet man Veränderungen, die für eine bestimmte Konservierungsart spezifisch sind, zum anderen gibt es wesentliche Unterschiede bei den einzelnen Obst- und Gemüsearten. Ein Vergleich der einzelnen Konservierungsmethoden muß neben der visuellen Beurteilung des konservierten Gutes auch die Qualität des tischfertigen Produktes in visueller, chemischer, physikalischer, physiologischer und anderer Hinsicht erfassen.

\section{Naßkonservierung}

Bei der Naßkonservierung geht ein großer Teil der Stoffe in Lösung. Man wird bei der Untersuchung trotzdem keine Nährstoffverluste feststellen, sofern man den Gesamtinhalt einer Dose (Material und Flüssigkeit) untersucht.

Der genannte Nachteil der Naßkonservierung tritt erst in Erscheinung, wenn man den Doseninhalt auf den Tisch bringt und das konservierte Material ohne die Flüssigkeit genießt. Außerdem werden bei der Naßkonservierung durch die Hitze die thermolabilen Vitamine weitgehend zerstört.

Wie unterschiedlich sich die einzelnen Arten bezüglich der Eignung für die Konservierung verhalten, sieht man beim Vergleich von Erbse und Spinat. Während die Erbse eine relativ hochwertige Tischqualität besitzt, ist der Spinat aus der Dose von ausgesprochen schlechter Qualität. Dieser Unterschied, der sich bei der Naßkonservierung von Erbse und Spinat zeigt, ist beim Tiefgefrieren nicht vorhanden.

Man hat versucht, durch Züchtung die Mängel, die die Sorten der einzelnen Gemüse- und Obstarten aufweisen, zu beseitigen. So ist es z. B. gelungen, Erbsen zu züchten, die nicht gelieren. Es ist aber nicht gelungen, Erbsen, Bohnen und Spinat zu züchten, die bei Naßkonservierung grün bleiben.

Bei Pfirsichen und Ananas hat man durch Sortenauswahl und Züchtung die Qualität des naßkonservierten Materials erheblich gesteigert.

\section{Tiefgefrieren}

Das Tiefgefrieren von Obst und Gemüse ist zu Anfang der 30er Jahre in Gang gekommen. Entscheidend hierbei ist, daß nicht mit hohen, sondern mit niedrigen Temperaturen gearbeitet wird und dabei viele Eigenschaften wesentlich besser erhalten bleiben als bei der Naßkonservierung.

Das Material wird einer Temperatur von $-20^{\circ} \mathrm{C}$ bis $-50^{\circ} \mathrm{C}$ ausgesetzt, wobei man ein möglichst schnelles Gefrieren anstrebt. Je niedriger die Temperatur und je kürzer die Zeit, desto kleiner sind die Eiskristalle und desto geringer sind die Zerreißungen der Zellwände. Dieses wirkt sich auf die Qualität der aufgetauten Produkte aus. Anschließend wird das Material bei $-20^{\circ} \mathrm{C}$ gelagert und auch mit $-20^{\circ} \mathrm{C}$ verteilt, d. h. man braucht eine Kühlkette von der Verarbeitung bis zum Verbraucher, die den Transport und die Lagerung teuer und anfällig macht.

Gemüse muß vor dem Tiefgefrieren blanchiert werden, weil trotz einer Lagerung bei $-20^{\circ} \mathrm{C}$ ohne Blanchierung durch enzymatische Tätigkeit Umsetzungen im Kühlgut vor sich gehen und zu einer Qualitätsminderung führen (4). Durch das Blanchieren (Kurzerhitzen auf $100^{\circ} \mathrm{C}$ ) werden die Enzyme inaktiviert.

Bei Erdbeeren wird durch das Tiefgefrieren bzw. wahrscheinlich durch das Wiederauftauen eine wesentliche Minderung des Geschmacks verursacht, während bei anderen Beerenobstarten diese Veränderung nicht zu verzeichnen ist.

\section{Gefriertrocknung}

Bei der Gefriertrocknung wird zunächst das Gut auf $-30^{\circ} \mathrm{C}$ bis $-60^{\circ} \mathrm{C}$ abgekühlt, dann in einem Vakuum das entstandene Eis durch Erhitzung zur Sublimation 
gebracht. Unter Sauerstoffabschluß wird das Material zweckmäßigerweise in einer Stickstoffatmosphäre gelagert. Dieses Konservierungsverfahren hat eine Reihe von Vorteilen:

1. Je nach Wassergehalt des Ausgangsmaterials hat das getrocknete Gut $\mathbf{1 0 - 4 0 \%}$ des ursprünglichen Gewichts. Hiermit sind erhebliche Transportvorteile verbunden.

2. Die Lagerung ist gegenüber dem Gefriergut erheblich billiger.

3. Die Erhaltung der Werteigenschaften ist in vielen Fällen wesentlich besser als bei der Naßkonservierung.

Es erhebt sich die Frage, ob bei der Gefriertrocknung außer dem Wasser auch andere Stoffe entfernt werden und ob es sich hierbei um für die Qualität positive oder negative Stoffe handelt. Hierüber weiß man bisher wenig, und es wird eines erheblichen Aufwandes an Forschungsarbeit bedürfen, um diese Fragen zu klären. Aus der Tatsache, daß z. B. Äpfel, Birnen, Aprikosen, Pflaumen und Weintrauben an der Luft getrocknet werden, kann man schließen, daß bei einer Gefriertrocknung u. U. gute Qualitäten zu erreichen sein müßten. Ganz allgemein wissen wir aber über die Wiederverwendung von Obst und Beerenobst nach der Gefriertrocknung noch wenig, und es wird notwendig sein, die Methoden der Wiederverwendung zu entwickeln. Die Züchtung von Spezialsorten für das Gefriertrocknen befindet sich naturgemäß noch im Anfangsstadium.

\section{Vergleich der verschiedenen 0bst- und Gemüsearten bezïglich ihrer Eignung für die Konservierung}

In Tab. 1 sind die verschiedenen Obst- und Gemüsearten aufgeführt, der Umfang ihrer Konservierung und die Qualität des konservierten Produktes bei Naßkonservierung, Tiefgefrieren und Gefriertrocknung angegeben. Bewußt haben wir uns auf diese drei Konservierungsarten beschränkt und lassen zunächst die anderen Arten außer acht (Marmelade, Konfitüre, Essigkonservierung u. a.). Ferner wird die Art der Wiederverwendung (kalt oder heiß) angegeben und ob das Frischgut nur im Laufe kurzer Zeitspannen oder das ganze Jahr über zur Verfügung steht.

Es fällt auf, daß sowohl beim Obst als auch beim Gemüse ganz wenige Kulturarten bei den einzelnen Konservierungsverfahren im Vordergrunde stehen. So wird z. B. beim Kernobst nur die Birne in großem Maße naßkonserviert - der Apfel nur als Apfelmus - während sie weder tiefgefroren noch gefriergetrocknet wird. Dasselbe gilt beim Steinobst für den Pfirsich und die Aprikosen, bei den Südfrüchten für die Ananas und beim Gemüse für den Spargel. Während es beim Kernobst und bei den Südfrüchten keine Arten gibt, die tiefgefroren werden, ist es beim Steinobst allein die Pflaume, die für dieses Konservierungsverfahren in Frage kommt. Das Beerenobst dagegen eignet sich für das Tiefgefrieren sehr gut. Brombeeren, Erdbeeren, Heidelbeeren, Himbeeren, Johannisbeeren und Preiselbeeren werden sowohl naßkonserviert als auch tiefgefroren. Es gibt unter den Gemüsearten Universalisten, wie z. B. die Bohne und die Erbse, die für alle drei Konservierungsarten gut geeignet sind, während z. B. der Spinat beim Tiefgefrieren und Gefriertrocknen eine gute Qualität, beim Naßkonservieren eine schlechte Qualität gibt. Der Champignon ist geeignet für Naßkonservierung und Gefriertrocknung, nicht aber für das Tiefgefrieren.

Beim Gemüse findet man eine Ưbereinstimmung zwischen ,heißer" Wiederverwendung und guter Eignung für die Gefriertrocknung. Man müßte annehmen, daß eine Konservierung nur dann interessant ist, wenn die Rohware nicht das ganze Jahr über zur Verfügung steht. Das Gegenteil ist in einzelnen Fällen der Fall, so z. B. bei den Obstarten bei Ananas und Mandarine und beim Gemüse beim Champignon. 
Tabelle 1. Mengen- und Qualitätsvergleich von Obst- und Gemüsekonserven bei verschiedenen Konservierungsarten

Zahlen (10) in ( ) $=$ Einfuhr, $\mathbf{M}=$ Menge, $\mathbf{Q}=$ Qualität

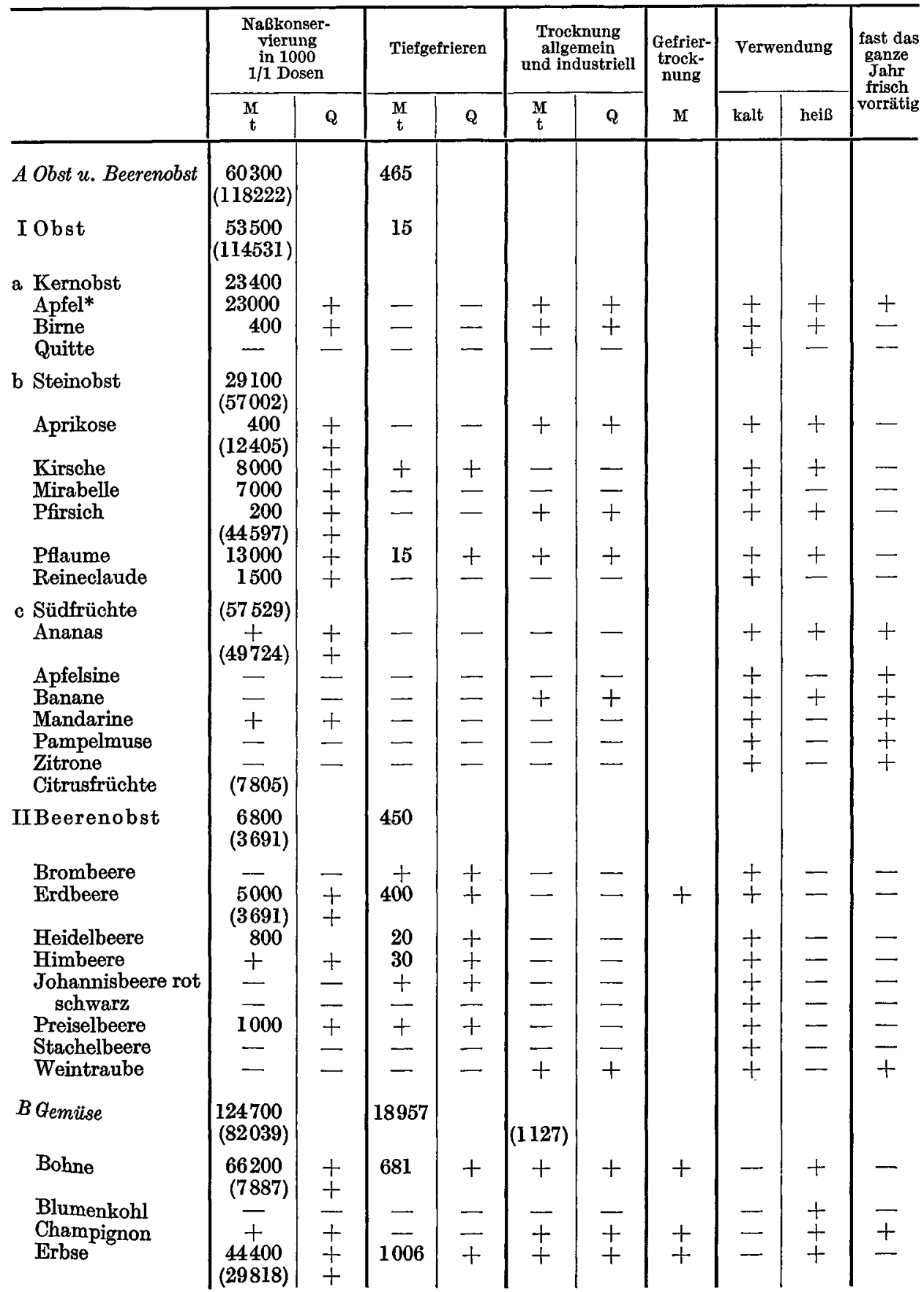


Tabelle 1 (Fortsetzung)

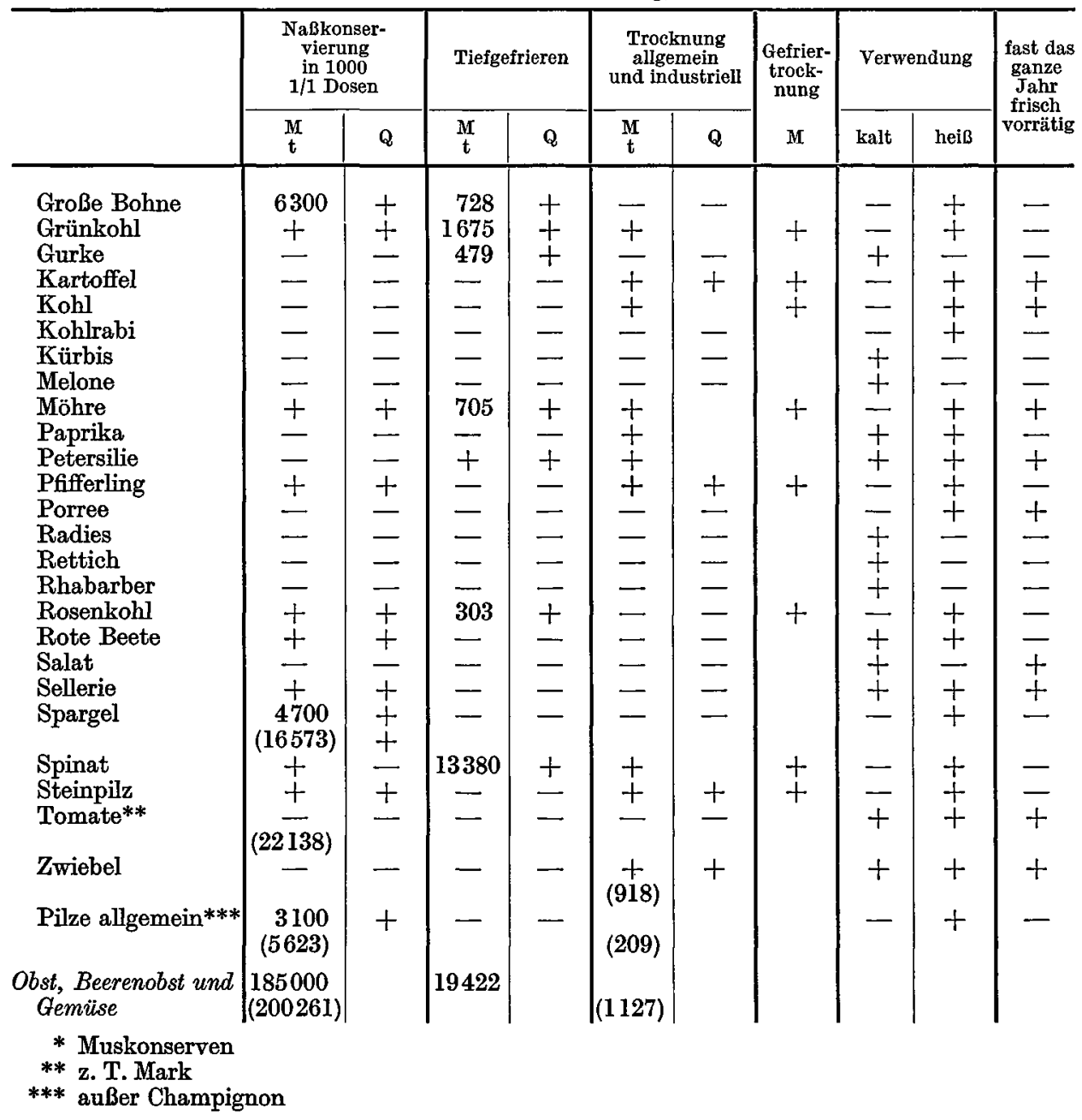

Beim Champignon, der praktisch das ganze Jahr über auf dem Frischmarkt zu haben ist, spielen die Naßkonservierung und neuerdings in den USA die Gefriertrocknung eine überragende Rolle.

\section{Besonderheiten der Gefriertrocknung}

Die Gefriertrocknung steht noch am Anfang ihrer technischen Entwicklung. Unsere Arbeiten sollen eigentlich klären, welche Aufgaben züchterischer Art im Zusammenhang mit der Gefriertrocknung von Obst und Gemüse zu erwarten sind. Hierzu ist es aber notwendig, zuerst Studien über die Technik der Gefriertrocknung und die Vor- und Nachteile gegenüber den anderen Konservierungsverfahren zu machen, weil wir glauben, daß keine reale Grundlage für die züchterische Bearbeitung von Obst und Gemüse hinsichtlich ihrer Eignung für die Gefriertrocknung vorhanden ist, solange man nicht die bestmögliche Art der Gefriertrocknung entwickelt hat. 


\section{Versuchsanlage}

Die Firma Christ hat nach unseren Angaben (Kallrstratos) eine Versuchsanlage gebaut, bei der die technologisch wesentlichen Parameter in weiten Grenzen variierbar sind:

1. Die Vorfriertemperatur und Geschwindigkeit.

2. Die Temperatur des Eiskondensators.

3. Die Temperatur des Trockengutes.

Die Werte können in ihrem Ablauf registriert werden. Außerdem besteht eine Möglichkeit der Messung der Leitfähigkeit im Gut, so daß man in der Lage ist, die Vorgänge entsprechend dem eutektischen Punkt des Gutes zu steuern. Die Anlage ist von uns in der Arbeit: „Die Anwendung von Pilot-Anlagen im Zusammenhang mit der Problematik der Gefriertrocknung von Lebensmitteln" (5) eingehend beschrieben worden. Außer den genannten Werten wird der Verbrauch an elektrischer Energie und an Kühlwasser durch entsprechende Vorrichtung gemessen. Hierdurch besteht die Möglichkeit, auch Aussagen über die Kosten und Rentabilität der Gefriertrocknung zu machen.

\section{Erhöhung der Rentabilität der Gefriertrocknung durch Aufteilung der einzelnen Vorgänge}

Wir haben uns bezüglich der Rentabilität der Gefriertrocknung Gedanken gemacht und sind zu dem Schluß gekommen, daß man die Wirtschaftlichkeit dadurch erhöhen könnte, daß man

a) das Vorfrieren separat in einem Kühltunnel vornimmt und damit die relativ teure Gefriertrocknungsanlage zeitlich entlastet,

b) die Vortrocknung bis zu einem bestimmten Restfeuchtigkeitsgehalt in der Hauptanlage durchführt,

c) die Nachtrocknung in einer separaten Nachtrockenkammer durchführt, von wo aus die Produkte nach Stickstoffbegasung ohne Kontakt mit dem atmosphärischen Sauerstoff der Verpackung zugeführt werden sollten.

Wir haben im eigenen Betrieb eine Trennung von Tiefgefrieren und Trocknen durchgeführt. Schon diese Trennung hat neben der Rentabilität folgende Vorteile:

1. Man wird bezüglich der Gefriertrocknung eines bestimmten Produktes unabhängig von der Erntezeit sein. Man kann zur Zeit der Ernte zunächst das gesamte Material einfrieren und anschließend nach Bedarf trocknen.

2. Die Trocknungsanlage wird durch die Trennung von Gefrieren und Trocknen wesentlich besser ausgenutzt. Es besteht hierdurch die Möglichkeit, unabhängig von der jeweiligen Erntezeit den Trocknungsapparat das ganze Jahr hindurch kontinuierlich in Betrieb zu halten, ihn aber, wenn es sich als zweckmäßig erweisen sollte, für eine einzige Kulturart einzusetzen. erhöht.

3. Die Kapazität der Gefriertrocknungsapparatur wird durch diese Arbeitsteilung wesentlich

\section{Qualitätsänderungen des konservierten Gutes durch die Geiriertrocknung}

Man hat festgestellt, daß beim Tiefgefrieren die Qualität des aufgetauten Gutes um so besser ist, je schneller das Einfrieren erfolgte (möglichst tiefe Temperaturen). Bei der Gefriertrocknung gibt es wesentlich mehr Faktoren, die man variieren kann, und die einen Einfluß auf die Qualität des Trockengutes haben können. Es ist aber zunächst notwendig, sich Gedanken darüber zu machen, was unter Qualität des Gutes zu verstehen ist, und wie man diese Qualität messen kann. Es wäre zu unterscheiden zwischen der äußeren Qualität (Farbe, Form) und der inneren Qualität (Geschmacksund Inhaltsstoffe) des Gefriertrocknungsproduktes. Von großer Bedeutung ist auch die Qualität, in der das Material auf den Tisch kommt. Unsere Untersuchungen wurden mit Champignons, Erdbeeren und Himbeeren begonnen.

\section{Schrumpfung der Rohware bei der Gefriertrocknung}

Bei Versuchen mit Champignons stellten wir fest, daß die Schrumpfung bei verschiedenen Trocknungsarten verschieden stark sein kann. Wir haben daher mit der Feststellung der Verringerung des Volumens des Trockenproduktes gegenüber dem Frischgut begonnen. $1000 \mathrm{ml}$ Champignons schrumpften bei einer normalen Trocknung bei $100^{\circ} \mathrm{C}$ auf etwa $100-150 \mathrm{ml}$ zusammen. 
Bei der Gefriertrocknung hatten wir zunächst Werte von $600 \mathrm{ml}$. Nachdem wir die einzelnen Vorgänge beim Gefriertrocknen variiert und in ihrem Einfluß auf das Volumen des gefriergetrockneten Gutes studiert hatten, erhielten wir im günstigsten Fall $700 \mathrm{ml}$, d. h. man kann durch Variieren der Einzelvorgänge bei der Gefriertrocknung die Schrumpfung verringern. Wir haben demnach eine Möglichkeit, durch die Messung der Veränderung des Volumens eine Aussage über die Qualität bezüglich der Formerhaltung zu machen. Außer dieser objektiv meßbaren Schrumpfung kann man die Schrumpfung auch an der Objektoberfläche direkt beobachten. Es besteht aber keine Möglichkeit, objektive Messungen in dieser Richtung durchzuführen.

\section{Stoffverluste bei der Gefriertrocknung}

Wir haben bei der Naßkonservierung gesehen, daß Stoffe aus dem Material in die umgebende Flüssigkeit austreten, d. h. im konservierten Material treten Stoffverluste ein. Es erhebt sich daher die Frage, ob auch bei der Gefriertrocknung mit ähnlichen Verlusten zu rechnen ist.

Bei chemischen Untersuchungen des Oxalatgehaltes des Spinats beobachteten wir, daß durch die Gefriertrocknung ein Verlust von Oxalat eintrat.

Wir untersuchten an den reinen Substanzen Oxalsäure, Natriumoxalat und Calciumoxalat die Veränderungen bei der Gefriertrocknung. Hierbei stellten wir eine völlige Gewichtsabnahme der Oxalsäure fest, während Natrium- und Calciumoxalat sich im Gewicht praktisch nicht veränderten (6).

Hieraus schlossen wir, daß Verluste von Oxalsäure bei der Gefriertrocknung auch beim Spinat eintreten können, und daß es die Oxalsäure ist, die in den oben beschriebenen Fällen verlorengegangen war. Ausgehend von den Oxalsäureverlusten beim Spinat haben wir die verschiedensten Stoffe und Stoffgruppen: Kohlenhydrate, Fette, Eiweiß, Vitamine, Aromastoffe, ätherische Öle, Alkohole, Aldehyde, Ester, organische Säuren, die im Pflanzenmaterial vorkommen, auf ihr Verhalten bei der Gefriertrocknung untersucht. Wir haben dieselben Stoffarten in ihrem Verhalten bei Zimmertemperatur $\left(20^{\circ} \mathrm{C}\right)$, bei $70^{\circ} \mathrm{C}$, bei $30^{\circ} \mathrm{C}$ und Vakuum 10 Torr und in der Gefriertrocknung bei $-30^{\circ} \mathrm{C}$ und $+30^{\circ} \mathrm{CTabulartemperatur} \mathrm{und} 0,2$ Torr, jeweils in 24 Std untersucht. Generell kann man sagen, daß unter den verschiedenen Trocknungsarten die größten Verluste bei Gefriertrocknung auftraten (7).

Bei Gefriertrocknung bleiben erhalten: Kohlenhydrate, Fette und Öle, Eiweiß und Vitamine. Es ist eine positive Eigenschaft der Gefriertrocknung, daß einige Vitamine nicht zerstört werden wie bei der Naßkonservierung.

Es gehen verloren: Aromastoffe, ätherische Öle, Alkohole, Aldehyde und Ester(7). Von den Säuren gehen die Monocarbonsäuren, z. B. Buttersäure und n-Capronsäure, verloren. Dicarbonsäuren, z. B. Oxalsäure, gehen langsam verloren, während Tricarbonsäuren, z. B. Citronensäure, erhalten bleiben.

Wir wollen die Säuren einer weiteren eingehenden Prüfung unterziehen, um festzustellen, ob diese Gesetzmäßigkeit bezüglich der Mono-, Di- und Tricarbonsäuren in dem beschriebenen Verhalten sich generell bestätigt.

Aus Ergebnissen, die wir bei reinen Substanzen und einigen Nahrungsmitteln erhalten hatten, kann man bestimmte Schlußfolgerungen ziehen: Zwiebeln, Lauch und andere verwandte Arten verlieren einen Teil der Aromastoffe, die ihren Wert ausmachen. Raps, Rübsen, Stoppelrüben und Kohl verlieren aller Voraussicht nach bei der Gefriertrocknung die negativ zu wertenden Geschmacksstoffe, so daß nach der Gefriertrocknung dieses Material sogar wertvoller ist als das frische Gut. Ester sind häufig Ursachen eines spezifischen Geschmacks bzw. Geruchs, so daß ihr Verlust 
sowohl positiv als auch negativ zu werten ist, je nachdem es sich um positive oder negative Geschmackseigenschaften der Ester handelt.

Es sei nur am Rande darauf hingewiesen, daB eine Reihe von tropischen und subtropischen Früchten ihres Terpentinölgehaltes wegen ungenießbar ist. Diese Früchte könnte man vielleicht durch Gefriertrocknung, d. h. Eliminierung des Terpentinöles, genießbar machen. Voraussetzung ist natürlich, daß in den Früchten außer dem Terpentinöl keine nicht sublimierenden, negativen Geschmacksstoffe enthalten sind.

Wir haben nicht nur die in Pflanzen vorkommenden Stoffe untersucht, sondern auch tierische Produkte und konnten auch bei diesen Veränderungen feststellen. Interessant ist die Gefriertrocknung von Butter (Deutsche Markenbutter), bei der außer Wasser auch noch zusätzlich andere Stoffe zu sublimieren scheinen, so daß beim Gefriertrockenen mehr als nur rund $15 \%$ des Gewichtes verlorengehen. Es wäre eine Sonderaufgabe zu prüfen, welche speziellen Stoffe bei der Gefriertrocknung von tierischen Produkten verlorengehen, damit man eine Ưbersicht darüber erhält, welche Qualitätsminderungen bei tierischen Erzeugnissen durch die Gefriertrocknung eintreten.

Unsere anschließenden Untersuchungen sollten zeigen, ob es möglich ist, durch entsprechende Änderungen im Ablauf der Gefriertrocknung zu verhindern, daß Wertstoffe verlorengehen und zu erreichen, daß Stoffe, die unerwünscht sind, verschwinden. Die ersten Untersuchungen auf diesem Gebiet haben gezeigt, daß keine wesentlichen Veränderungen der stofflichen Verluste zu erreichen sind. Diese Untersuchungen sind jedoch nicht abgeschlossen.

\section{Unterschiede der Rehydration, d. h. Wiederaufnahme von Wasser nach der Gefriertrocknung}

Wir kommen jetzt zu der Frage „Qualität auf dem Tisch“. Naßkonservierte Champignons verlieren durch die Auslaugung in heißem Wasser lösliche Stoffe, Aromastoffe und Wirkstoffe; sie schrumpfen und verlieren auch an äußerer Qualität. Es sind also bereits vor der weiteren Zubereitung erhebliche Mengen an wertvollen Stoffen verlorengegangen. Leider liegen keine Messungen über die Qualitätsminderung bei aus naßkonservierten Champignons zubereiteten Speisen vor.

Bei gefriergetrocknetem Champignongut fügt man in der Regel nicht so viel Wasser zu, wie ursprünglich den Pilzen entzogen worden ist. Dies ist die Ursache, weshalb aus gefriergetrockneten Champignons zubereitete Speisen aromatischer und wohlschmeckender sind als die aus frischen und insbesondere aus naßkonservierten Champignons zubereiteten (Konzentrationseffekt).

Die äußere Form-, Farb- und Stofferhaltung ist keineswegs das alleinige Maß für die ,Tischqualität". Wir haben gesehen, daß gefriergetrocknete Zwiebeln ihre Wertstoffe verloren haben und dadurch trotz schönsten Aussehens, ,wertlos" geworden sind. Es gibt aber auch noch eine andere Ursache dafür, daß konserviertes Material, das als Konserve erstklassig aussieht und vollwertig erscheint, für den Tisch wertlos ist. Ein naßkonservierter Pfirsich kommt nach Öffnung der Dose in der Form auf den Tisch, in der er in der Dose vorhanden war. Das gefriergetrocknete Obst muB, wenn es als Kompott genossen werden soll, zunächst durch Zuckerwasser-Aufnahme genießbar gemacht werden. Die Qualität wird an dem tischfertigen Produkt beurteilt.

Es ist möglich, Kompott sowohl aus tiefgefrorenen als auch aus gefriergetrockneten Erdbeeren auf den Tisch zu bringen. Die tiefgefrorenen aufgetauten Beeren weisen ganz allgemein - obwohl es ganz erhebliche Sortenunterschiede gibt - einen gegenüber der Frischfrucht veränderten Geschmack auf. 
Die aus gefriergetrockneten Erdbeeren hergestellten Kompotte haben das Säurearoma der Frischfrucht. Sie zeigen gegenüber dem Kompott aus tiefgefrorenen Erdbeeren eine wesentlich bessere Qualität und keinen „Gefriergeschmack“".

Aus diesen Versuchen ist abzuleiten, daß das Tiefgefrieren der Beeren noch keine Veränderung des Geschmacks bedingt und daß die Veränderung erst beim Auftauen eintritt. In ähnliche Richtung weist die Beobachtung, daß Speiseeis, das aus tiefgefrorenen Beeren hergestellt wird, ohne daß ein Auftauen der Beeren erfolgt, besonders gut schmeckt und keinen Tiefgefriergeschmack aufweist. Man sollte versuchen, Methoden des Wiederauftauens von tiefgefrorenen Erdbeeren zu entwickeln, bei denen man das Entstehen des Gefriergeschmacks vermeidet. Es ist darauf hinzuweisen, daß Himbeeren und die meisten anderen Beerenobstarten im Gegensatz zu Erdbeeren diesen Gefriergeschmack nicht bekommen.

Vorläufig ist noch unbekannt, welche wahrscheinlich chemischen Veränderungen während des Auftauens diese Geschmacksunterschiede bedingen. Festgestellt haben wir in unserem Institut, daB der Ascorbinsäuregehalt von tiefgefrorenen und gefriergetrockneten Erdbeeren gleich ist (8). Wir haben weiter festgestellt, daß bei tiefgefrorenen und wiederaufgetauten und gefriergetrockneten Erdbeeren Unterschiede bezüglich des Gehalts an einzelnen Zuckerarten bestehen. Es ist aber nicht anzunehmen, daB diese Unterschiede im Zucker den Unterschied im Geschmack erklären.

Beim Gemüse, das ja generell zum Tischfertigmachen aufgekocht, erhitzt oder geschmort wird, liegen die Qualitätsmerkmale der tischfertigen Produkte sehr günstig. Der Vorteil der Gefriertrocknung, geringes Gewicht der Ware beim Einkauf, müßte sich auswirken können. Die Hausfrau fügt das verlorene Wasser zum Tischfertigmachen zu, erhitzt das Material und erhält ein qualitativ hochwertiges Produkt. Diese Ausführungen gelten für Bohnen, Erbsen, Spinat und Pilze aller Art.

Es ist anzunehmen, daß die Gemüsearten, die den Brassica-Arten nahestehen, eine besondere Annehmlichkeit haben werden. Ihnen wird der Kohlgeruch, der bei der Verarbeitung von frischem und tiefgefrorenem Kohl eintritt, fehlen. Es kann sein, daß dieser Vorteil dem gefriergetrockneten Kohl eine Monopolstellung verschaffen wird.

In der Tab. 2 haben wir diejenigen Kulturarten zusammengestellt, die ätherische Öle (Lauchöle $=\mathrm{L}$, Senföle $=\mathrm{S}$, andere ätherische Öle $=\mathrm{A}$ ) als Inhaltsstoffe saftiger Pflanzenteile enthalten. (Gemüse, Gewürzpflanzen, Heilpflanzen, Obst). Ferner ist angegeben, ob es sich um erwünschte $(+)$ oder unerwünschte (-) Bestandteile handelt.

Von den aufgeführten Pflanzenarten werden die einen durch Verluste der ätherischen Öle an Qualität gewinnen, die anderen an Qualität verlieren. Es wäre eine technische Aufgabe, die dem Material entzogenen Stoffe wiederzugewinnen. Die Gewinnung ist einmal als Sublimat zusammen mit dem Wasser möglich, zum anderen getrennt vom Wasser in den Stoffen, zu denen die einzelnen Stoffe eine besonders hohe Affinität besitzen.

\section{Züchtung von für das Gefriertrocknen geeigneten Sorten}

Man kann der Tab. 1 entnehmen, welche Konservierungsverfahren für die einzelnen Kulturarten in Frage kommen. Der Züchter, der sich mit der Steigerung der Eignung von Kulturpflanzen für ein bestimmtes Konservierungsverfahren beschäftigt, wird sich zunächst der Pflanzenarten annehmen, die der Menge nach im Vordergrunde stehen. Er wird speziell die Eigenschaften bearbeiten, von denen die gute Konservierbarkeit abhängt. 
Die Tatsache, daß beim Tiefgefrieren und Wiederauftauen von Erdbeeren wesentliche Wertminderungen gegenüber der Frischqualität bezüglich Form-, Farb- und Geschmackserhaltung auftreten, hatte 1942 dazu geführt, daß wir eine planmäßige Züchtung von Erdbeersorten aufnahmen, mit dem Ziel, Sorten zu züchten, die eine gute Form-, Farb- und Geschmackserhaltung aufweisen.

Tabelle 2. Ätherische Öle als Inhaltsstoffe saftiger Pflanzenteile. Zeichenerklärung: L = Lauchöle, $\mathrm{S}=$ Senföle, $\mathrm{A}=$ andere ätherische Öle und ihre Bestandteile, $+=$ erwünscht, $-=$ nicht erwünscht

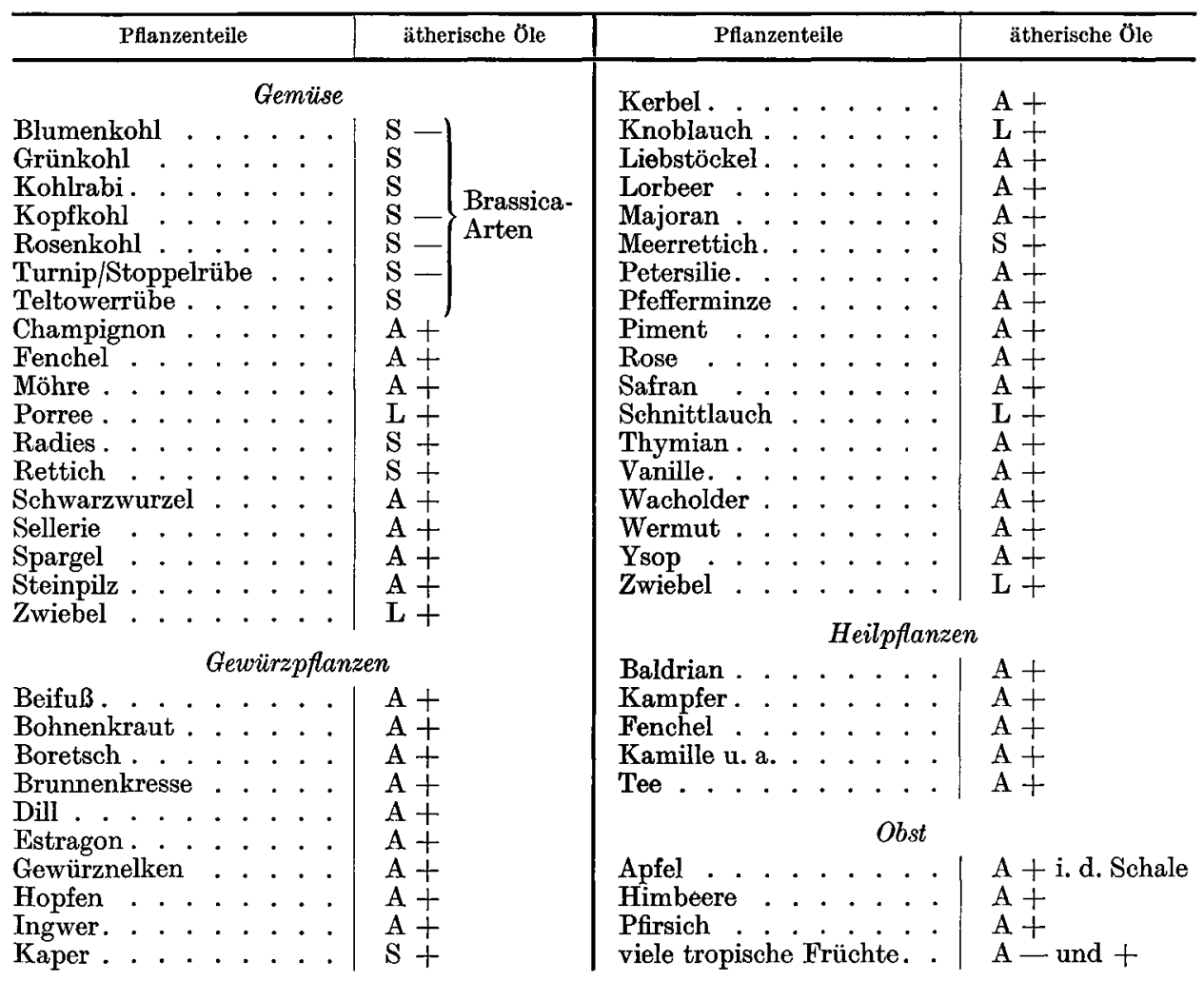

Das Ergebnis dieser Arbeit war eine Reihe von Senga-Sorten: Senga 29, SengaSengana u. a., deren Früchte in der genannten Richtung eine gute Gefriereignung besitzen. Für den gleichen Zweck wurde die Züchtung von Erbsensorten aufgenommen. Auch bei den Erbsen wurden gute Fortschritte bezüglich der Gefriereignung erzielt. Es gelang die Kombination einer guten Geschmacksqualität mit einer hervorragenden, leuchtenden, dunkelgrünen Farbe.

\section{Aufstellung der Zuchtziele}

Wir stehen heute vor der Frage, die Zuchtziele aufzustellen, die sich aus der Anwendung der Gefriertrocknung ergeben (9). Diese Ziele müssen auf Realisierbarkeit an Hand der weiter oben beschriebenen Ergebnisse überprüft werden. 


\section{Oxalatarmer Spinat}

Bei der Züchtung eines oxalatarmen Spinats muß in Rechnung gestellt werden, daß die Oxalsäure beim Gefriertrocknen verschwindet und daß man sich daher in der Hauptsache mit der Eliminierung aller löslichen Oxalate, auch des Kalium-, Natriumoxalates, befassen müßte. Dieses ist notwendig, weil unsere physiologischen Tierversuche gezeigt haben, daß gelöste Oxalate im Urin in Erscheinung treten, während das Calciumoxalat vermutlich über den Darm (Kot) ausgeschieden wird. Unsere Oxalatuntersuchungen haben gezeigt, daß es nur geringe Sortenunterschiede gibt.

Hieraus ist aber nicht zu schließen, daß die Züchtung oxalatarmer Spinate unmöglich wäre. Wir glauben, daß durch eine Individualauslese in Kombination mit einer planmäßigen züchterischen Bearbeitung des Pflanzenmaterials oxalatarme, insbesondere an löslichen Oxalaten arme, Spinate gezüchtet werden könnten.

\section{Formerhaltung beim Champignon}

Im Rahmen unserer Gefriertrocknungsversuche nimmt der Champignon eine Sonderstellung ein. An ihm wurde die volumetrische Methode der Qualitätsbestimmung entwickelt. Es wurde festgestellt, daß sowohl volumetrisch meßbare Unterschiede in bezug auf die Schrumpfung bei Champignonsorten als auch sichtbare Schrumpfungsunterschiede der Oberfläche vorhanden sind (Abb. 1), d. h. man kann

1. durch Variation der Gefriertrocknungstechnik eine optimale Trocknung und

2. durch Auslese innerhalb der Art eine Steigerung der äußeren Qualität erreichen.

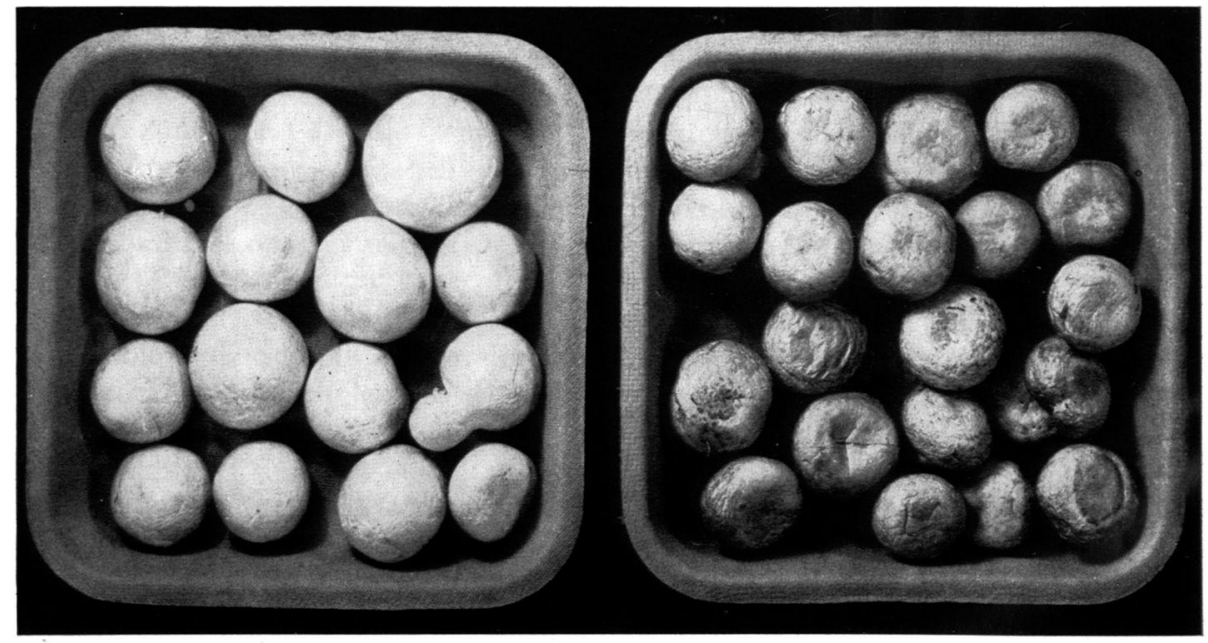

a

b

Abb. 1 a u. b. Unterschiedliches Verhalten zweier Champignonsorten bei gleicher Gefriertrocknung. a) Keine Oberflächenschrumpfung. b) Starke Oberflächenschrumpfung

\section{Erdbeeren mit guter Form- und Geschmackserhaltung nach der Rehydration}

Bei der Erdbeere konnten wir Sortenunterschiede bezüglich der Wiederverwendbarkeit nach der Gefriertrocknung feststellen. Von den von uns geprüften Sorten Senga-Sengana, Senga-Precosa und Senga-Gigana erwies sich allein die Senga-Sengana nach der Gefriertrocknung für die Wiederverwendung geeignet. Sie eignet sich für die Herstellung von Kompott und kandierten Früchten, während die beiden anderen 
Senga-Sorten (Precosa und Gigana) wegen eines ungenügend festen Gerüstes die notwendige Formerhaltung vermissen lassen (Abb. 2). Die Ergebnisse machen es wahrscheinlich, daß man Sorten züchten kann, die die Senga-Sengana in bezug auf Festigkeit und damit Formerhaltung und auf Geschmack übertreffen.

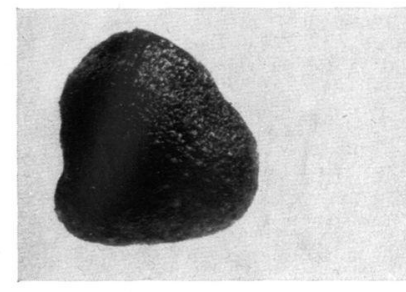

a

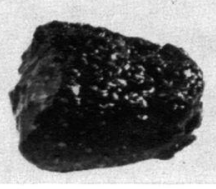

b

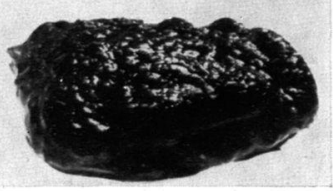

c

Abb. 2a-c. Unterschiedliche Wiederaufnahme von Flüssigkeit bei 3 verschiedenen Erdbeersorten und gleicher Art der Trocknung. a) Senga-Sengana: gut, b) Senga-Precosa u. c) Senga-Gigana: schlecht, wegen starker Schrumpfung

\section{Auslese der durch Gefriertrocknung an Wert gewinnenden Arten}

Wir besitzen somit die ersten Anhaltspunkte dafür, daß man die Gefriereignung von Obst und Gemüse auch auf züchterischem Wege verbessern kann. Wir werden aus der Fülle der Obst- und Gemüsearten diejenigen auslesen müssen, die eine praktische Bedeutung für die Gefriertrocknung besitzen, und diese werden wir dann in züchterische Bearbeitung nehmen. Darüber hinaus werden wir aber auch feststellen, welche Arten allein durch die Gefriertrocknung an Wert gewinnen.

Wir haben weiter oben Angaben über die Verluste von ätherischen Ölen, Estern, Säuren und Vitaminen gemacht. Es wäre z. B. einerseits beirn Kohl unter Berücksichtigung dieser Ergebnisse nicht notwendig, ,senföl"-freie Sorten zu schaffen, andererseits wäre es müßig, durch Steigerung des Gehalts an ätherischen ölen oder an Estern bei bestimmten Arten den Geschmack zu erhöhen, weil diese Stoffe bei der Gefriertrocknung zum Teil verloren gehen und dadurch das tischfertige Material keine Qualitätserhöhung durch eine derartige Steigerung zeigte. Wir brauchen diese Pflanzenarten nur bezüglich der Werteigenschaften in züchterische Bearbeitung zu nehmen, die von der Gefriertrocknung unbeeinflußt bleiben.

\section{Zusammenfassung.}

In der vorliegenden Arbeit wird eine Übersicht über die Probleme der Gefriertrocknung im Zusammenhang mit der Lebensmittelkonservierung dargestellt. Die neue Methode wird mit den schon vorhandenen Konservierungsarten verglichen, hauptsächlich mit der Naßkonservierung und dem Tiefgefrieren. Zum eingehenden Studium über die Eignung verschiedener Obst- und Gemüsearten wurde eine PilotAnlage verwendet. Einige Zuchtziele bei Kulturpflanzen im Zusammenhang mit der Gefriertrocknung werden angedeutet.

\section{Literatur}

1. Neumann, K.: Grundriß der Gefriertrocknung. 2. Aufl. Göttingen, Frankfurt, Berlin: Musterschmidt.

2. Neumann, K.: Fortschritte der Methodik und Anwendung der Gefriertrocknung. Chem. Ing. Techn. 29, 267 (1957).

3. Hackenberg, V.: Umschau 14, 428 (1961).

4. Hodge, J.: J. agric. Food Chem. 15, 928 (1953).

5. Kallistratos, G., R. v. Sengbusch, K. Goergen: Diese Z. im Druck. 
6. Kallistratos, G., u. R. v. Sengbusch: Naturwissen. 51, 367 (1964).

7. Kallistratos, G., u. R. v. Sengbusch: Nutr. et diät (im Druck).

8. SÜCKeR, I.: Persönliche Mitteilung, 1964.

9. v. SENGBUSCH, R.: 5. Gefriertrocknungstagung, Köln 14 (1962).

10. Nehrina, P., u. H. Krause: Konserventechn. Taschenbuch. S. 1138, 1140, 1141, 1152. Braunschweig: G. Hempel 1963. 